\title{
An early superficial non-ampullary duodenal tumor cured with endoscopic submucosal dissection: A case report
}

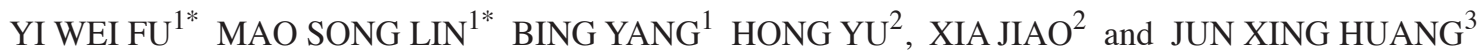 \\ Departments of ${ }^{1}$ Gastroenterology, ${ }^{2}$ Pathology and ${ }^{3}$ Oncology, Taizhou People's Hospital, \\ Taizhou, Jiangsu 225300, P.R. China
}

Received May 13, 2016; Accepted May 19, 2017

DOI: $10.3892 / \mathrm{ol} .2017 .6576$

\begin{abstract}
Early superficial non-ampullary duodenal tumors are particularly rare, the clinical manifestations, including typical endoscopic or imaging features, and treatment methods are not well-characterized. The present case report describes a case of an asymptomatic 74-year-old male who presented to the Taizhou People's Hospital (Taizhou, China) for a regular health screening, where a primary superficial non-ampullary duodenal tumor was identified. Upper endoscopy revealed $\sim 1.2 \mathrm{~cm}$ lesion in the second portion of the duodenum. Chromoscopy and magnification endoscopy indicated an early cancer characteristic. Subsequent endoscopic submucosal dissection was performed to remove the lesion. Histopathology validated that the lesion was a high-grade intro-epithelial neoplasm without lymph node or blood vessel invasion.
\end{abstract}

\section{Introduction}

Superficial non-ampullary duodenal tumor (SNADT), adenoma or carcinoma, is a rare type of gastrointestinal tract epithelial tumor and can be defined as a lesion limited to the mucosa, and/or submucosa which is not arising from the papilla of Vater $(1,2)$. Typically, the likelihood of developing duodenal adenoma in patients with familial adenomatous polyposis (FAP) is high and this type of adenoma may progress to carcinoma (3). In contrast, sporadic NADT may develop de novo or through the adenoma-carcinoma pathway, as observed in

Correspondence to: $\mathrm{Dr}$ Mao Song Lin, Department of Gastroenterology, Taizhou People's Hospital, 210 Yingchun Road, Taizhou, Jiangsu 225300, P.R. China

E-mail:1ms0605@163.com

Dr Jun Xing Huang, Department of Oncology, Taizhou People's Hospital, 210 Yingchun Road, Taizhou, Jiangsu 225300, P.R. China E-mail: hjxtz@sina.cn

*Contributed equally

Key words: superficial non-ampullary duodenal tumor, narrow band imaging, magnifying endoscopy, high grade intro-epithelial neoplasm, endoscopic submucosal dissection patients with FAP (4). The prognosis of patients with advanced stage carcinoma is poor (5). However, if the duodenal tumor is diagnosed at an early stage and the patient undergoes complete resection, using an endoscopic method, the patient may experience a markedly improved outcome. Therefore, early detection and treatment of lesions are required (6).

As the incidence of sporadic SNADT is rare, endoscopic markers suggestive of early stage SNADT have not been established. In addition, although endoscopic approaches, including endoscopic mucosa resection (EMR) and endoscopic submucosal dissection (ESD), are minimally invasive, and localized treatments in comparison to conventional surgery, there are a number of high risk complications (including bleeding and perforation) which may occur during the endoscopic resection (ER) (7-9). Therefore, the relatively rare incidence, and the presence of a thin duodenal wall and rich vascularity make it difficult to detect and treat SNADT lesions.

The present case report describes an elderly patient presented to the Taizhou People's Hospital (Taizhou, China) for esophagogastroduodenoscopy and was identified to exhibit a superficial lesion $(\sim 1.2 \mathrm{~cm})$ in the second portion of the duodenum. Chromoscopy and magnification endoscopy with narrow band imaging (ME-NBI) indicated an early stage lesion. Subsequently, ESD was performed to remove the lesion. Histopathology validated the high-grade intro-epithelial tumor diagnosis with negative margins, and without lymph or blood vessel involvement.

\section{Case report}

In the present case report, a 70-year-old Chinese male presented to the Taizhou People's Hospital (Taizhou, China) for a regular health screening and was diagnosed with an early NADT following a gastroduodenoscopy exam. Pathological examination validated this diagnosis, following the removal of the lesion by ESD. On admission, the patient's resting blood pressure was normal, at $120 / 80 \mathrm{mmHg}$, the patient had no significant family medical history and exhibited no symptoms, including melena, abdominal pain, weight loss, or dyspepsia. The results of physical examination were unremarkable. Laboratory results were normal, as follows: White blood cell count, 7,530 cells $/ \mathrm{mm}^{3}$ with $66.5 \%$ neutrophils; hemoglobin level, $15.0 \mathrm{~g} / \mathrm{dl}$; mean corpuscular volume, $90.0 \mathrm{fl}$; mean corpuscular hemoglobin concentration, $33.4 \mathrm{~g} / \mathrm{dl}$; and platelet count, 
189,000 cells $/ \mathrm{mm}^{3}$. Liver and renal function, and electrolyte levels were all identified to be normal. Tests for tumor markers, including carcinoembryonic antigen and carbohydrate antigen 19-9, revealed no abnormalities; however, the carbohydrate antigen 72-4 was mildly increased. The fecal occult blood test results were negative. A colonoscopy exam revealed only a sporadic polyp, which excluded the possibility of FAP. Abdominal computed tomography did not identify a duodenal mass or any enlargement of the lymph nodes in the abdomen.

Following an upper endoscopy exam, a red flat lesion was identified $(\sim 1.2 \mathrm{~cm})$, which was elevated with a slightly depressed area and located on the second portion of the duodenum. The mass was edematous and extended laterally along the side opposite the ampulla. The tumor was soft and it bled easily at the touch (Fig. 1A). In order to overcome the limitation of traditional white-light endoscopy, ME-NBI was used to identify distinctions between potentially malignant lesions. The surface pattern of the mass was preserved in one region and was absent in another region, which may indicate a mixed type of tumor (10). In addition, irregular vascular patterns with clear demarcation were observed (Fig. 1B), which indicated a category 4/5 tumor associated with high-grade dysplasia (HGD) or intramucosal carcinoma, according to the revised Vienna classification (11).

Considering the age of the patient and the characteristic of the identified lesion, the patient's family members were consulted and written informed consent was obtained to remove the lesion through endoscopy. Forcep biopsy was not performed to avoid associated fibrosis, which may affect additional endoscopic treatment. ESD as a total biopsy was initially performed. If histology from the ESD had revealed the tumor was confined to the mucosal layer, and the horizontal and vertical margin was negative, the patient may have achieved remission and subsequently subjected to follow-up. However, if histology had identified that the tumor exhibited submucosal invasion or lymphovascular involvement, or the margin was positive, additional treatments, including a partial resection or pancreaticoduodenectomy with lymphadenectomy, may have been considered.

Briefly, a soft transparent hood (D-201-13404) was attached to the tip of the gastroscope with water jet functions (GIF-Q260J) and a $\mathrm{CO}_{2}$ insufflation system (UCR) (all from Olympus Corporation, Tokyo, Japan) was used during the whole process. A high frequency electrosurgical generator model VIO300D (Erbe Elektromedizin GmbH, Tubingen, Germany) was used, which was set at a cutting current (Endocut mode, effect $2,40 \mathrm{~W}$ ) for mucosal incision and at a coagulating current for submucosal dissection (Swift Coagulation mode, effect $4,40 \mathrm{~W}$ ). Firstly, the margin of the duodenal tumor was marked according to the chromoendoscopy using indigo carmine dye spraying. Following submucosal injection of sodium hyaluronate (Bausch and Lomb Freda Pharmaceutical Co., Ltd., Shangdong, China), containing 0.005 and $0.0025 \%$ indigo carmine, and epinephrine, respectively, a mucosal incision was made outside the tumor margin using a Dual knife (KD-650Q; Olympus Corporation) (Fig. 1C). A direct dissection of the submucosal layer was performed to obtain the complete specimen and subsequently, a complete en bloc resection was accomplished (Fig. $1 \mathrm{C}$ and D). The lesion was removed and visible vessels located in the bottom of the ulcer were clamped using hemostatic forceps (FD-410LR; Olympus Corporation). The surface of the wound was closed using three titanium clips (Micro-Tech Co., Ltd., Nanjing, China). The pathological results validated the HGD with negative horizontal and vertical margins using hematoxylin and eosin staining (Fig. 1E). The patient was administered a proton pump inhibitor (PPI) for 1 week, and was observed in hospital in case of delayed complications, including bleeding and perforation. The patient was discharged and transited to follow-up.

\section{Discussion}

The small intestine accounts for $75 \%$ of the entire digestive tract and tumors which arise from it constitutes $\sim 5 \%$ of all gastrointestinal (GI) tract tumors. However, the duodenum, which only accounts for $4 \%$ of the small intestine, has a relatively higher proportion of associated tumors compared with tumors of the jejunum and ileum. Furthermore, although accounting for only $0.3 \%$ of malignant digestive tract tumors, duodenal carcinoma constitutes $50 \%$ of all malignant small intestinal tumors (12). Owing to the rarity, non-specific signs and symptoms, and the fact that the duodenum is typically overlooked during upper gastrointestinal endoscopy, duodenal tumors pose diagnostic difficulties. Almost all non-ampullary neoplasms are incidentally discovered during routine endoscopy, as was the case in the present report.

The exact etiology of duodenal tumors remains unknown. Previous studies have demonstrated that patients with FAP, Crohn's disease, celiac disease, Lynch syndrome or Peutz-Jeghers syndrome exhibit an increased risk of developing duodenal carcinoma $(13,14)$. In the present case report, no associations with the aforementioned diseases were identified and therefore this lesion appeared a sporadic duodenal tumor. It was identified that patients with a duodenal carcinoma exhibited a 5-year survival rate of $<30 \%$ and the prognosis of patients with an advanced stage was even poorer $(5,15)$. However, if such lesions are identified at an early stage and subsequently treated through endoscopic curative resection or operation, the outcome for patients is often markedly improved (16). It is important to identify duodenal tumors at an early stage; however, due to the lack of specific clinical manifestations, and the fact that the duodenum is typically overlooked during endoscopy exam, this rare type of lesion is difficult to identify at an early stage. Furthermore, a definition for early NADC has not been established according to the depth of tumor invasion and the risk of lymph node metastasis (17). Previous studies have used the regulations for early colorectal or gastric cancer $(18,19)$ and for tumor invasion into the lamina propria, muscularis mucosa (T1a), or submucosa (T1b), neglecting lymph node metastasis (20). It is easier to determine the margins of early duodenal flat lesion using endoscopy examination than to distinguish the depth of tumor infiltration, for example T1a from T1b duodenal cancer. However, developments in endoscopic technology (including high-resolution endoscopy and image-enhanced endoscopy) and the establishment of a series of criteria, enabling the identification of GI tumors (the Vienna classification), have allowed for the identification of early superficial duodenal tumor lesions $(9,11)$. In addition, these developments enable duodenal tumor lesions to be resected without operation $(9,11)$. 

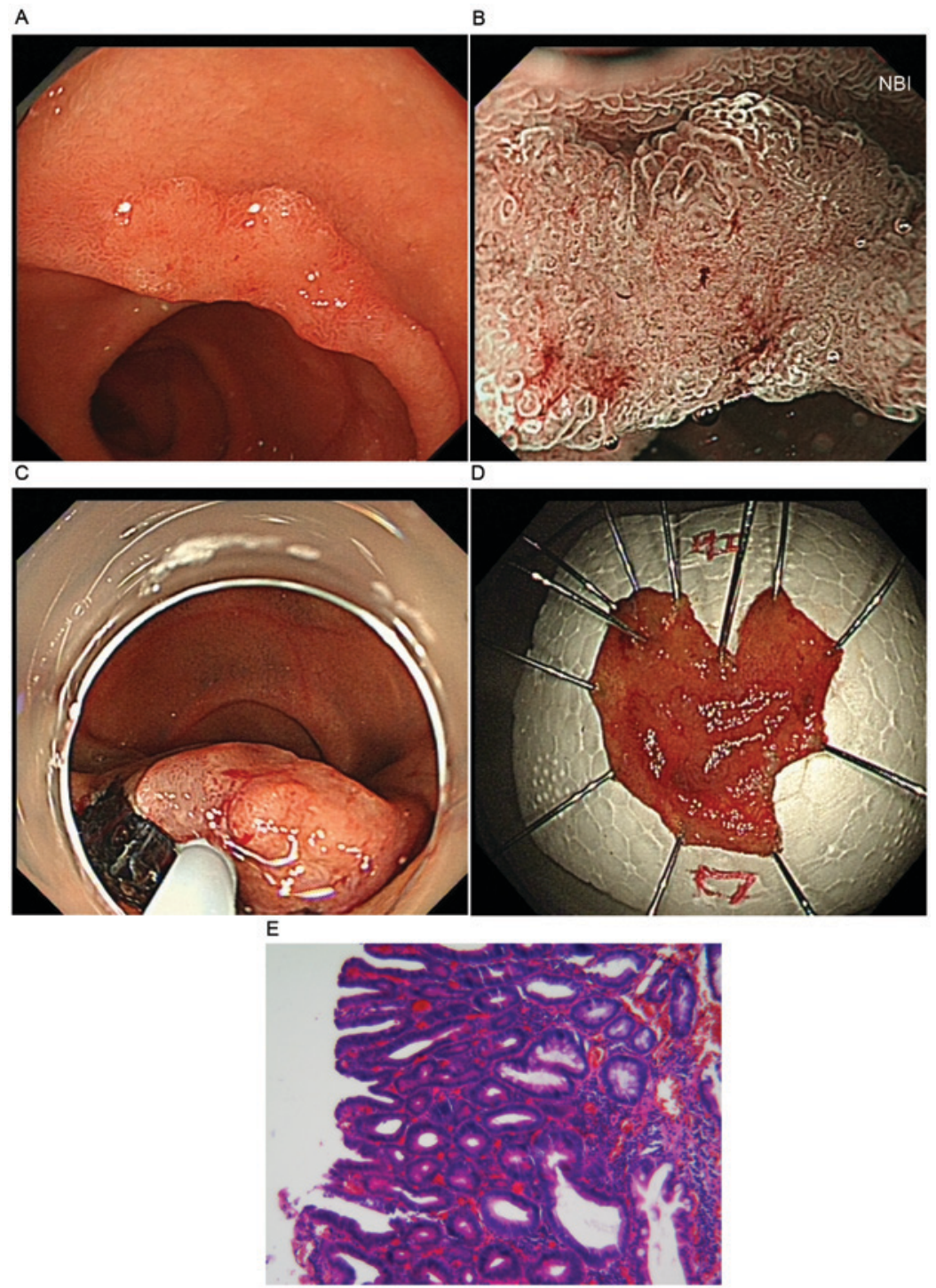

Figure 1. An early superficial non-ampullary duodenal tumor. (A) A flat elevated lesion (1.2 cm) was visible in the second portion of the duodenum, using conventional endoscopy with white light imaging. (B) Surface pattern was preserved in one region and is absent in another region, and an unclassified vascular pattern was exhibited following magnifying endoscopy with narrow band imaging. (C) The endoscopic submucosal dissection method was performed to cure the lesion. (D) The whole lesion was cut from duodenum. (E) The lesion was diagnosed as a category $4 / 5$ tumor by hematoxylin and eosin staining (magnification, $\mathrm{x} 100$ ).

To overcome the limitations of traditional white-light endoscopy with chomoendoscopy, ME-NBI may be used to identify the potential malignant lesion. NBI refers to an imaging technique for endoscopic diagnostic medical tests, where light of specific blue and green wavelengths is used to enhance the details of certain aspects of the mucosa surface (21). ME-NBI has been revealed to enhance visualization of the micromucosal and microcirculatory structure for a detailed assessment of the early lesions, and may be used to differentiate digestive tract lesions more accurately compared with conventional endoscopy $(22,23)$. However, there are a limited number of studies where ME-NBI has been used in association with SNADT. In 2006, Uchiyama et al (24) reported that ampullary polyps may be classified, using NBI, as: Type I, oval-shaped villi; type II, pinecone/leaf-shaped villi; or type III, irregular/non-structured. A previous study categorized the surface pattern of SNADT as preserved, micrified or absent and subsequently divided these into two types, mono and mixed, using a novel diagnostic algorithm for ME-NBI (10). In addition, vessels were defined to possess one of the following patterns: Absent, network, intrastructural vascular (ISV) with dilated, tortuous in the mucosal structure or unclassified (10). All mixed-type lesions identified were classified as category 4 (mucosal high-grade neoplasia) or category 5 (submucosal invasion by carcinoma) tumors, on the basis of the revised Vienna classification (10). Additionally, $\sim 50 \%(10 / 23)$ of the monotype lesions were identified to be category 3 (mucosal low-grade neoplasia) tumors (10). However, among the monotype lesions, the proportion of category $4 / 5$ tumors was $100 \%$ in lesions with an unclassified vascular pattern, $64.3 \%$ in lesions with an ISV pattern, $33.3 \%$ in lesions with an absent pattern, and $25.5 \%$ (1/4) in lesions with a network pattern (10). Thus, the results of this study are useful for the identification of benign and malignant lesions in SNADTs (10). Furthermore, a multicenter study revealed that a significantly higher number of high-grade dysplasia 
or superficial adenocarcinomas compared with low-grade dysplasia were identified in tumors with a diameter $>5 \mathrm{~mm}$ with solely or predominantly red coloration (9). According to the aforementioned categorical descriptions, the lesion in the present study was identified to possess a mixed type surface with an unclassified pattern vessel and may be a category $4 / 5$ tumor.

Comprehensive observation of the surface and vascular patterns of SNADT, using enhanced endoscopy, and ME-NBI classification maybe used to perform histological diagnosis of a lesion. However, owing to the limited number of SNADT cases, the additional advantages of magnifying endoscopy in early duodenal cancer remain unknown and additional studies are required. Furthermore, due to the thin wall of the bowel, unintended fibrosis induced by common biopsy may affect subsequent ER, and the diagnostic accuracy of enhanced endoscopy and biopsy has been identified to be similar (78 and $75 \%$, respectively) $(9,25)$. Therefore, in the present case report, the ESD procedure was performed to remove the lesion for pathological analysis instead of forcep biopsy.

The conventional treatment methods for duodenal tumor are local surgical excision or radical surgery, which are both characterized by significant proportions of recurrence and relatively higher morbidity and mortality compared with endoscopy therapy (26). Surgical resection is invasive, it is difficult to determine the site and extent of the lesion from outside the intestine, and resect it locally. Endoscopic resection for the treatment of SNADT is less invasive, and a number of endoscopic resection techniques, including snare polypectomy, EMR, ESD and APC ablative methods for treatment of SNADT are available. However, the endoscopic therapeutic methods for SNADT are not standardized, and clinicians are required to determine whether EMR or ESD may be used on the basis of the size and location of the lesion, and the operability of patients. Small duodenal adenomas maybe removed en bloc by conventional EMR; however, ESD is a more reliable option, as the removal of this type of malignant tumor is difficult using conventional EMR. ESD is recognized as a promising minimally invasive approach, which is curative and safe, even for large lesions in the stomach, esophagus, and colon (27-29). In contrast, performing ESD in the duodenum is technically challenging, due to the distinct anatomical characteristics of the duodenum, and has not been accepted as a radical method of local resection until the present moment (30). There have been a limited number of studies on the use of ESD to treat of SNADTs; however, a complete resection rate between 80 and $100 \%$ with no recurrence was revealed $(17,31)$. In the present case report, although the patient was discharged with no complications, there has been a relatively high proportion of bleeding and perforation identified in duodenal ESD, attributed to the extensive second-order arterial blood supply, and thin wall of the duodenum (17,31-33). Duodenal ESD may be performed with caution in selected patients to avoid serious complications and follow-up is required.

Previous studies have demonstrated that if the perforation is in the course of the surgery, the hole maybe closed using an endoscopic titanium clip or sutured using anylon snare, followed by the administration of antibiotics and fasting for several days $(8,34-37)$. Abdominal computed tomography may be performed in all patients with perforation to identify whether the retroperitoneal perforation has occurred. If the perforation cannot be closed and the ESD procedure cannot be performed, the patient may undergo surgery. In addition, hematemesis or melena, caused by delayed post-surgical bleeding, requires additional endoscopy and a hemostatic procedure, using hemostatic forceps, or clips, similar to performing a resection. All patients are recommended to be administered a PPI for $\geq 2$ months following ESD, and undergo follow-up endoscopic examinations 2 and 6 months following ESD, and subsequently every 12 months. In addition, abdominal computed tomography or ultrasonography may be performed annually to identify lymph node and distant metastases, if the final pathological diagnosis of the specimen revealed characteristics of malignancy $(8,34-37)$.

The present case report identified and diagnosed an early SNADT using ME-NBI endoscopy, which was completely removed. Additional studies with a larger number of cases are required to acquire information on the diagnostic and treatment of SNADT, using endoscopy.

\section{Acknowledgements}

The present case report was supported by the Project of Health Department of Jiangsu Province (China) (grant no. H201363), the Social Development Project of Taizhou City (Jiangsu, China) (grant no. TS025) and the Project of Jiangsu University (2014).

\section{References}

1. Alwmark A, Andersson A and Lasson A: Primary carcinoma of the duodenum. Ann Surg 191: 13-18, 1980.

2. Yokoyama T, Saito D, Kondo H, Kido M, Hosokawa K, Shirao K, Yokota T, Yamaguchi H, Oguro Y, Ishikawa T, et al: Endoscopic diagnosis of malignant lesions of the duodenum. Stomach Int 28: 641-649, 1993.

3. Vasen HF, Möslein G, Alonso A, Aretz S, Bernstein I, Bertario L, Blanco I, Bülow S, Burn J, Capella G, et al: Guidelines for the clinical management of familial adenomatous polyposis (FAP). Gut 57: 704-713, 2008.

4. Perzin KH and Bridge MF: Adenomas of the small intestine: A clinicopathologic review of 51 cases and a study of their relationship to carcinoma. Cancer 48: 799-819, 1981.

5. Bakaeen FG, Murr MM, Sarr MG, Thompson GB, Farnell MB, Nagorney DM, Farley DR, van Heerden JA, Wiersema LM, Schleck CD and Donohue JH: What prognostic factors are important in duodenal adenocarcinoma? Arch Surg 135: 635-641, 2000.

6. Lim CH and Cho YS: Nonampullary duodenal adenoma: Current understanding of its diagnosis, pathogenesis and clinical management. World J Gastroenterol 22: 853-861, 2016.

7. Lépilliez V, Chemaly M, Ponchon T, Napoleon B and Saurin JC: Endoscopic resection of sporadic duodenal adenomas: An efficient technique with a substantial risk of delayed bleeding. Endoscopy 40: 806-810, 2008.

8. Ono H, Nonaka S, Uedo N, Kaise M, Oyama T, Doyama $\mathrm{H}$, Kokawa A, Kaneko K, Kodashima S, Tanabe S, et al: Clinical issues of duodenal EMR/ESD. Stomach Int 46: 1669-1677, 2011.

9. Goda K, Kikuchi D, Yamamoto Y, Takimoto K, Kakushima N, Morita Y, Doyama H, Gotoda T, Maehata Y and Abe N: Endoscopic diagnosis of superficial non-ampullary duodenal epithelial tumors in Japan: Multicenter case series. Dig Endosc 26 (Suppl 2): S23-S29, 2014.

10. Kikuchi D, Hoteya S, Iizuka T, Kimura R and Kaise M: Diagnostic algorithm of magnifying endoscopy with narrow band imaging for superficial non-ampullary duodenal epithelial tumors. Dig Endosc 26 (Suppl 2): S16-S22, 2014.

11. Dixon MF: Gastrointestinal epithelial neoplasia: Vienna revisited. Gut 51: 130-131, 2002.

12. Kerremans RP, Lerut J and Penninckx FM: Primary malignant duodenal tumors. Ann Surg 190: 179-182, 1979. 
13. Heniford BT, Iannitti DA, Evans P, Gagner M and Henderson JM: Primary nonampullary/periampullary adenocarcinoma of the duodenum. Am Surg 64: 1165-1169, 1998.

14. Dabaja BS, Suki D, Pro B, Bonnen M and Ajani J: Adenocarcinoma of the small bowel: Presentation, prognostic factors, and outcome of 217 patients. Cancer 101: 518-526, 2004.

15. Howe JR, Karnell LH, Menck HR and Scott-Conner C: The American College of Surgeons Commission on Cancer and the American Cancer Society. Adenocarcinoma of the small bowel: Review of the National Cancer Data Base, 1985-1995. Cancer 86 : 2693-2706, 1999.

16. Yoshimura N, Goda K, Tajiri H, Ikegami M, Nakayoshi T and Kaise M: Endoscopic features of nonampullary duodenal tumors with narrow-band imaging. Hepatogastroenterology 57: 462-467, 2010.

17. Kakushima N, Ono H, Takao T, Kanemoto H and Sasaki K: Method and timing of resection of superficial non-ampullary duodenal epithelial tumors. Dig Endosc 26: 35-40, 2014.

18. Japanese Society for Cancer of the Colon and Rectum: Japanese Classification of Colorectal Carcinoma. 8th edition. Kanehara Shuppan, Tokyo, pp9-10, 2013.

19. Japanese Gastric Cancer Association: Japanese Classification of Gastric Carcinoma. 14th edition. Kanehara Shuppan, Tokyo, pp7-8, 2010.

20. Takahashi T, Ando T, Kabeshima Y, Kawakubo H, Shito M, Sugiura $\mathrm{H}$ and Omori T: Borderline cases between benignancy and malignancy of the duodenum diagnosed successfully by endoscopic submucosal dissection. Scand J Gastroenterol 44: $1377-1383,2009$.

21. Gono K, Obi T, Yamaguchi M, Ohyama N, Machida H, Sano Y, Yoshida S, Hamamoto Y and Endo T: Appearance of enhanced tissue features in narrow-band endoscopic imaging. J Biomed Opt 9: 568-577, 2004.

22. Nakanishi H, Doyama H, Takemura K, Yoshida N, Tsuji K, Takeda Y, Asahina Y, Kito Y, Ito R, Hayashi T, et al: Detection of pharyngeal cancer in the overall population undergoing upper GI endoscopy by using narrow-band imaging: A single-center experience, 2009-2012. Gastrointest Endosc 79: 558-564, 2014.

23. Miwa K, Doyama H, Ito R, Nakanishi H, Hirano K, Inagaki S, Tominaga K, Yoshida N, Takemura K, Yamada S, et al: Erratum to: Can magnifying endoscopy with narrow band imaging be useful for low grade adenomas in preoperative biopsy specimens? Gastric Cancer 18: 446, 2015.

24. Uchiyama Y, Imazu H, Kakutani H, Hino S, Sumiyama K, Kuramochi A, Tsukinaga S, Matsunaga K, Nakayoshi T and Goda K, et al: New approach to diagnosing ampullary tumors by magnifying endoscopy combined with a narrow-band imaging system. J Gastroenterol 41: 483-490, 2006.

25. Kakushima N, Kanemoto H, Sasaki K, Kawata N, Tanaka M, Takizawa K, Imai K, Hotta K, Matsubayashi H and Ono H: Endoscopic and biopsy diagnoses of superficial, nonampullary, duodenal adenocarcinomas. World J Gastroentero 21: 5560-5567, 2015 .
26. Farnell MB, Sakorafas GH, Sarr MG, Rowland CM, Tsiotos GG, Farley DR and Nagorney DM: Villous tumors of the duodenum: Reappraisal of local vs. extended resection. J Gastrointest Surg 4: 13-21, 2000.

27. Yahagi N, Fujishiro M, Kakushima N, Kobayashi K, Hashimoto T, Oka M, Iguchi M, Enomoto S, Ichinose M, Niwa H and Omata M: Endoscopic submucosal dissection for early gastric cancer using the tip of an electrosurgical snare (thin type). Dig Endosc 16: 34-38, 2004.

28. Oyama T, Tomori A, Hotta K, Morita S, Kominato K, Tanaka M and Miyata Y: Endoscopic submucosal dissection of early esophageal cancer. Clin Gastroenterol Hepatol 3 (7 Suppl 1): S67-S70, 2005

29. Yamamoto H, Yahagi N and Oyama T: Mucosectomy in the colon with endoscopic submucosal dissection. Endoscopy 37: 764-768, 2005

30. Hoteya S, Yahagi N, Iizuka T, Kikuchi D, Mitani T, Matsui A, Ogawa O, Yamashita S, Furuhata T, Yamada A e al: Endoscopic submucosal dissection for nonampullary large superficial adenocarcinoma/adenoma of the duodenum: Feasibility and long-term outcomes. Endosc Int Open 1: 2-7, 2013.

31. Matsumoto S, Miyatani H and Yoshida Y: Endoscopic submucosal dissection for duodenal tumors: A single-center experience. Endoscopy 45: 136-137, 2013

32. Takeuchi M, Kobayashi M, Shioji K, Togashi T, Hashimoto S, Sato Y, Narisawa R and Aoyagi Y: Prevention and management of complications in endoscopic resection for non-ampullary duodenal neoplasms (in Japanese with an English abstract). Endosc Digest 22: 1561-1158, 2010.

33. Honda T, Yamamoto H, Osawa H, Yoshizawa M, Nakano H, Sunada K, Hanatsuka K and Sugano K: Endoscopic submucosal dissection for superficial duodenal neoplasms. Dig Endos 21: 270-274, 2009

34. Doyama H, Tominaga K, Yoshida N, Takemura K and Yamada S: Endoscopic tissue shielding with polyglycolic acid sheets, fibrin glue and clips to prevent delayed perforation after duodenal endoscopic resection. Dig Endos 26 (Suppl 2): 41-45, 2014.

35. Takimoto K, Imai Y and Matsuyama K: Endoscopic tissue shielding method with polyglycolic acid sheets and fibrin glue to prevent delayed perforation after duodenal endoscopic submucosal dissection. Dig Endosc 26 (Suppl 2): 46-49, 2014.

36. Inoue $\mathrm{T}$, Uedo $\mathrm{N}$, Yamashina $\mathrm{T}$, Yamamoto $\mathrm{S}$, Hanaoka $\mathrm{N}$, Takeuchi Y, Higashino K, Ishihara R, Iishi H, Tatsuta M, et al: Delayed perforation: A hazardous complication of endoscopic resection for non-ampullary duodenal neoplasm. Dig Endos 26: 220-227, 2014.

37. Mori H, Shintaro F, Kobara H, Nishiyama N, Rafiq K, Kobayashi M, Nakatsu T, Miichi N, Suzuki Y and Masaki T: Successful closing of duodenal ulcer after endoscopic submucosal dissection with over-the-scope clip to prevent delayed perforation. Dig Endosc 25: 459-461, 2013. 